\title{
Perineurial Cell
}

National Cancer Institute

\section{Source}

National Cancer Institute. Perineurial Cell. NCI Thesaurus. Code C41442.

A cell that belongs to the supporting tissue surrounding a nerve fiber bundle. It has thin long bipolar cytoplasmic processes, pinocytotic vesicles, fragments of external lamina and/or external lamina-like material, attachment plaques, and desmosome-like junctions. 